\title{
On-line Multi-attributes Procurement Combinatorial Auctions Bidding Strategies
}

\author{
Jian Chen and He Huang \\ School of Economics and Management, Tsinghua University, Beijing, 100084, China \\ jchen@mail.tsinghua.edu.cn \\ huangh02@mails.tsinghua. edu.cn
}

\begin{abstract}
Based on the work of Krishna and Rosenthal (1996) about combinatorial auctions bidding equilibrium analysis and Che's (1993) research about one-unit multi-attributes auctions, we construct a multi-attributes procurement combinatorial auction (MAPCA) for 2 objects, through a first-score, sealed-bid format. There are two kinds of bidders: $n$ simple bidders and $m$ diverse bidders considered in this model. With some assumptions, we finally obtain the equilibrium bidding strategies for the both two kinds of bidders.
\end{abstract}

\section{Introduction}

Combinatorial auctions have been applied in a variety of environments involving economic transactions, and they have the potential to play an important role in electronic procurement transactions for supply chain management. Examples are Net Exchange (www.nex.com) who procures transportation services for Sears Logistics (Ledyard et al. 2002), procurement combinatorial auctions for truck services at Home Depot (Elmaghraby and Keskinocak 2003), and IBM's procurement combinatorial auction for Mars Incorporated (Hohner et al. 2003). The characteristics of combinatorial auctions that we will treat in the procurement context are items involving multiple attributes. Comparing with normal combinatorial auctions, the property of multiple attributes is somewhat unique in procurement auctions. When an auctioneer procures items by auctions, she may allow the potential suppliers to bid for various products bundles, which is called Procurement Combinatorial Auctions (CAs).

The topics on one-unit auctions' bidding strategies and winning probability are abundantly addressed. However, discussions about CAs' bidding strategies are inadequate. Krishna and Rosenthal (1996) considered situations where multiple objects are auctioned simultaneously by means of a second-price sealed-bid auction, and derived the bidding equilibrium strategies for the synergy bidder. Rosenthal and Wang (1996) studied a simultaneous-auction model with synergies, common values, and constructed strategies. Che (1993) studies design competition in one item procurement by developing a model of two-dimensional (price and quality) auctions. The purpose of this paper is to discuss bidding strategies in a specific multi-attributes procurement CA setting. 


\section{The Model}

In this model, there are 2 identical objects to be procured by a first-score sealed-bid auction. And two kinds of bidders, i.e. simple bidder and diverse bidder are considered. Each simple bidder is interested in only one object, while each diverse bidder, say diverse bidder $j$, is interested in these two objects with synergy effect. For each object, there are $n$ interested simple bidders. We can also regard the situation as two identical auctions started in two separated rooms; each room has $n$ simple bidders, and diverse bidders are apt to bid in both rooms. Each bidder has a privately known supply cost function for the object. Other assumptions are as follows.

Bidding vector is $\{p, q\} \in R_{+}{ }^{2}, p, q$ denote bidding price and quality-bid separately. Especially, quality-bid, in our model, is a unified number to measure all non-price factors of the objects. The scoring function of the bidding vectors has a quasi-linear form

$$
S(q, p)=s(q)-p
$$

where $s($.$) is strictly increasing with respect to q$.

Number of simple bidders, for each object, is $n$; number of diverse bidders is $m$.

The cost function of simple bidder $i$ is $c\left(q, \theta_{i}\right) . \theta_{i}$ denotes type of simple bidder $i$, which is private information of bidder $i$, and $\theta_{i}$ is drawn from interval $[\underline{\theta}, \bar{\theta}]$ with identical distribution $F($.$) , which is common knowledge. c(.,$.$) is increasing with$ respect to $q$ and $\theta_{i}$. Type of diverse bidder $j$ is denoted as $\theta_{j}$ which is drawn from interval $\left[\underline{\theta}_{g}, \bar{\theta}_{g}\right]$ with the identical distribution $F_{g}(.) ; \theta_{j}$ is also private information. The cost function of the diverse bidder is $c_{g}\left(q, \theta_{j}\right)$ for each object. $\left[\underline{\theta}_{g}, \bar{\theta}_{g}\right]$ and $F_{g}($.$) are common knowledge; c_{g}(.,$.$) is increasing with respect to q$ and $\theta_{j}$.

If winning, profit of simple bidder $i$, with bid $\{p, q\} \in R_{+}{ }^{2}$, is

$$
\pi_{i}(q, p)=p-c\left(q, \theta_{i}\right) .
$$

Profit of the diverse bidder $j$, with bid $\{p, q\} \in R_{+}^{2}$, is

$$
\left\{\begin{array}{l}
p-c_{g}\left(q, \theta_{j}\right), \quad \text { if win one } \\
2 p-2 c_{g}\left(q, \theta_{j}\right)+\alpha_{j}, \quad \text { if win both }
\end{array} .\right.
$$

where $\alpha_{j}$, i.e., synergy effect, is a non-negative constant which is diverse bidder $j$ 's private information.

With type $\theta_{i}$, simple bidder $i$ 's bidding strategy is formula (4): [Riley and Samuelson, 1981; Che, 1993; Chen and Huang 2004] 


$$
\left\{\begin{array}{l}
q\left(\theta_{i}\right)=\arg \max \left[s(q)-c\left(q, \theta_{i}\right)\right], \\
p\left(\theta_{i}\right)=c\left(q, \theta_{i}\right)+\int_{\theta_{i}}^{\bar{\theta}} c(q(t), t)\left[\frac{1-F(t)}{1-F\left(\theta_{i}\right)}\right]^{n} d t
\end{array} .\right.
$$

Diverse bidders know each other's profit function form, but do not know other diverse bidders' synergy effect value, say $\alpha_{j}$, which are drawn from $[0, \bar{\alpha}]$, and their identical distribution $F_{\alpha}($.$) is common knowledge. The model constructed above is$ called MAPCA in this paper.

\section{Bidding Strategy Analysis}

For simple bidders, Let $S_{0}\left(\theta_{i}\right) \equiv \max \left[s(q)-c\left(q, \theta_{i}\right)\right], \forall \theta_{i} \in[\underline{\theta}, \bar{\theta}]$. By using envelope theorem, $S_{0}$ (.) is a strictly decreasing function, therefore its inverse function exits. Let $v_{i} \equiv S_{0}\left(\theta_{i}\right), v_{i}$ can be regarded as scoring capability of simple bidder $i$. Let $H\left(v_{i}\right) \equiv 1-F\left(S_{0}^{-1}\left(v_{i}\right)\right)$, then distribution $F($.$) of \theta_{i}$ is transformed into distribution $H($. $)$ of $v_{i}$. Let $b \equiv S\left(q_{s}\left(\theta_{i}\right), p\right)$, where $q_{s}\left(\theta_{i}\right)=\arg \max \left[s(q)-c\left(q, \theta_{i}\right)\right]$. Obviously, $b$ is the score evaluated by the bidding of simple bidder with type $\theta_{i}$. Let $B($.$) denote$ the scoring function with the independent variable $v_{i}$, and $B($.$) is strictly increasing$ with $v_{i}$. Define $G(.) \equiv H\left(B^{-1}().\right)$ and $L(b)=\left\{H\left(B^{-1}(b)\right)\right\}^{n}$ for convenience. Intuitively, $L(b)$ is the probability of all $\mathrm{n}$ simple bidders who are interested in one of the two items get score less than $b$.

Similarly, for diverse bidders, let $S_{g}\left(\theta_{j}\right) \equiv \max \left[s(q)-c_{g}\left(q, \theta_{j}\right)\right], \forall \theta_{j} \in\left[\underline{\theta}_{g}, \bar{\theta}_{g}\right]$. By envelope theorem, $S_{g}($.$) is a strictly decreasing function, therefore its inverse func-$ tion exits. Let $v \equiv S_{g}\left(\theta_{j}\right), v$ is scoring capability of diverse bidder $j$. Let $H_{g}(v) \equiv 1-F_{g}\left(S_{0}^{-1}(v)\right)$, then distribution $F_{g}($.$) of \theta_{j}$ is transformed into distribution $H_{g}$ (.) of $v$, which also implies that the one-to-one mapping relationship from $v$ to $\theta$. Let $b \equiv S\left(q_{s}\left(\theta_{j}\right), p\right)$, where $q_{s}\left(\theta_{j}\right)=\arg \max \left[s(q)-c_{g}\left(q, \theta_{j}\right)\right]$, and $b$ is the score of the bidding by diverse bidder with type parameter $\theta_{j}$. Let $B_{g}(v)$ denote the scoring function with $v$, and $B_{g}($.$) is strictly increasing with v$. Define $G_{g}(.) \equiv H_{g}\left(B_{g}{ }^{-1}().\right)$ and $K(b) \equiv\left\{H_{g}\left(B_{g}^{-1}(b)\right)\right\}^{m-1}$. Actually, $K(b)$ is the probability of all other $m-1$ diverse bidders get score less than $b$. Note that $p-c\left(q_{s}\left(\theta_{j}\right), \theta_{j}\right)=v-b$, and then we have the expected revenue of any diverse bidder with type $\theta_{j}$ who bids quality-bid $q_{s}$ and price $p$ as follows, 


$$
\begin{aligned}
& \pi\left(\left(q_{s}, p\right) \mid \theta_{j}\right) \\
& =L^{2}\left(s\left(q_{s}\right)-p\right) \times K\left(s\left(q_{s}\right)-p\right) \times\left(2(p-c)+\alpha_{j}\right) \\
& \quad+2 L\left(s\left(q_{s}\right)-p\right) \times\left(1-L\left(s\left(q_{s}\right)-p\right)\right) \times K\left(s\left(q_{s}\right)-p\right) \times(p-c)
\end{aligned}
$$

where, $q_{s}$ represents quality bidding, $p$ is price bidding, $c$ denotes the corresponding cost with quality bidding $q_{s}$. Now, we would show an important proposition (lemma 1) to illustrate any diverse bidder with any specific type, say $\theta_{j}$, will report qualitybid to maximize $s(q)-c_{g}\left(q, \theta_{j}\right)$.

LEMMA 1. The diverse bidder with type $\theta_{j}$ follows the bidding strategy in which quality-bid $q_{s}\left(\theta_{j}\right)$ is

$$
q_{s}\left(\theta_{j}\right)=\arg \max \left[s(q)-c_{g}\left(q, \theta_{j}\right)\right] .
$$

Proof: Assume there exists another equilibrium strategy $(q, p)$, and $q \neq q_{s}\left(\theta_{j}\right)$, then construct a bid $\left(q^{\prime}, p^{\prime}\right)$,

$$
\text { where } q^{\prime}=q_{s}\left(\theta_{j}\right), \quad p^{\prime}=p+s\left(q_{s}\left(\theta_{j}\right)\right)-s(q) \text {. }
$$

Note $\quad S\left(q^{\prime}, p^{\prime}\right)=S(q, p)=S$.

Then, with bid $\left(q^{\prime}, p^{\prime}\right)$, the expected revenue of diverse bidder is below,

$$
\begin{aligned}
\pi\left(\left(q^{\prime}, p^{\prime}\right) \mid \theta_{j}\right) \\
=L^{2}(S) K(S)\left[2\left(p+s\left(q_{s}\left(\theta_{j}\right)\right)-s(q)-c_{g}\left(q^{\prime}\right)\right)+\alpha_{j}\right] \\
\quad+2 L(S)(1-L(S)) K(S)\left(p+s\left(q_{s}\left(\theta_{j}\right)\right)-s(q)-c_{g}\left(q^{\prime}\right)\right) \\
=L^{2}(S) K(S)\left\{2 \times\left[\left(p-c_{g}(q)+\left(s\left(q_{s}\left(\theta_{j}\right)\right)-c_{g}\left(q_{s}\left(\theta_{j}\right)\right)-\left(s(q)-c_{g}(q)\right)\right)\right]+\alpha_{j}\right\}\right. \\
\quad+2 L(S)(1-L(S)) K(S)\left[\left(p-c_{g}(q)+\left(s\left(q_{s}\right)-c_{g}\left(q_{s}\left(\theta_{j}\right)\right)-\left(s\left(q_{1}\right)-c_{g}(q)\right)\right)\right]\right.
\end{aligned}
$$

Note $q_{s}\left(\theta_{j}\right)=\arg \max \left[s(q)-c_{g}\left(q, \theta_{j}\right)\right]$, therefore,

$$
\pi\left(\left(q^{\prime}, p^{\prime}\right) \mid \theta_{j}\right) \geq L(S) K(S) \cdot\left\{L(S)\left[2\left(p-c_{g}(q)\right)+\alpha_{j}\right]+2(1-L(S)) \cdot\left(p-c_{g}(q)\right)\right\}
$$

Thus, we have

$$
\pi\left(\left(q^{\prime}, p^{\prime}\right) \mid \theta_{j}\right) \geq \pi\left((q, p) \mid \theta_{j}\right)
$$

This completes the proof.

Without loss of generality, let $s\left(q_{s}\left(\theta_{j}\right)\right)=1$; from LEMMA 1 and (5), we know the optimization problem of the diverse bidder with type $\theta_{j}$ can be expressed as follows: 


$$
\begin{aligned}
& \max _{p} \pi\left(\left(q_{s}, p\right) \mid \theta_{j}\right) \\
& \quad=\max _{p}\left\{L(1-p) \cdot K(1-p) \cdot\left[\left(L(1-p) \cdot\left(2(p-c)+\alpha_{j}\right)+2(1-L(1-p)) \cdot(p-c)\right]\right\}\right. \\
& \quad=\max _{p}\left\{\alpha_{j} L^{2}(1-p) K(1-p)+2 L(1-p) K(1-p)(p-c)\right\}
\end{aligned}
$$

where $c$ denotes the corresponding cost of the diverse bidder with her equilibrium quality-bid $q_{s}\left(\theta_{j}\right) ; c$ is also the optimal cost of the bidder. Actually, $q_{s}\left(\theta_{j}\right)$ and $c$ are determined by type $\theta_{j}$ uniquely. Let $l()=.L^{\prime}(),. k()=.K^{\prime}($.$) , by first derivative of$ (12) with respect to $p$, we have

$$
\begin{aligned}
\frac{d \pi}{d p} & =c-p-L(1-p) \cdot \frac{\alpha_{j} \cdot l(1-p) \cdot K(1-p)+\alpha_{j} \cdot L(1-p) \cdot k(1-p) / 2-K(1-p)}{l(1-p) \cdot K(1-p)+L(1-p) \cdot k(1-p)} \\
& =0
\end{aligned}
$$

Now considering the relationship between the optimal cost $c$ of diverse bidder with $\theta_{j}$ and her price bid $p$, from (13) we have

$$
c=p+L(1-p) \cdot \frac{\alpha_{j} \cdot l(1-p) \cdot K(1-p)+\alpha_{j} \cdot L(1-p) \cdot k(1-p) / 2-K(1-p)}{l(1-p) \cdot K(1-p)+L(1-p) \cdot k(1-p)} .
$$

Actually, (14) is the correspondence from optimal cost $c$ to possible optimal price bid $p$, and we can abstractly express (14) as $c=c_{p}(p)$. The only special feature used in the argument is that $c_{p}($.$) is convex. Convexity of c_{p}($.$) is not easy to characterize in$ terms of the primitive assumptions of our model. However, if $G_{g}($.$) and G($.$) are$ uniform distribution, then the function $c_{p}($.$) is indeed convex. If c_{p}($.$) is not convex,$ the situation becomes more complicated and worth pursuing in the future correlated works. Therefore, if $c_{p}($.$) is convex, then there exit at most 2$ roots of equation (14).

Corresponding to different optimal cost $c$, denote $p_{g}(c)$ as the solutions of (14). Define $p_{g}{ }^{*}(c)$ as the unique root or greater one of the two roots, i.e.,

$$
p_{g}{ }^{*}(c)=\left\{\begin{array}{l}
p_{g}(c), \text { for unique solution } \\
\max \left\{p_{g}(c)\right\}, \text { for othercase }
\end{array} .\right.
$$

By convexity of $c_{p}($.$) , the derivative of d \pi / d p$ is non-positive at the point of the greater root. That means the second derivative of expected revenue with respect to $p$, at the greater root, is non-positive. It is the second-order sufficient condition for local maximizer. Consequently, we are sure that $p_{g}{ }^{*}(c)$ is the local maximizer, while the smaller root is not. 
More specific, let $\bar{\alpha}=2$, that means $\alpha_{j} \in[0,2]$. And consider the excessive case in which the diverse bidder $j$ 's cost is 0 and $\alpha_{j}=2$, for individual rationality, the price bidding of her will not less than -1 . Therefore we assume the domain of $p$ is $[-1,1]$. Assume $G_{g}($.$) and G($.$) are all uniform distributions, then G_{g}()=.(1-p) / 2$, $G()=.(1-p)$. Correspondingly, we have

$$
\begin{gathered}
K(1-p)=((1-p) / 2)^{m-1}, p \in[-1,1], L(1-p)=(1-p)^{n}, p \in[0,1], \\
k(1-p)=[(1-m) / 2] \cdot[(1-p) / 2]^{m-2}, l(1-p)=-n \cdot(1-p)^{n-1} .
\end{gathered}
$$

Note that, when $p \in[-1,0]$, the diverse bidder will definitely surmounts all simple bidders with probability 1 . Then (12) can be rewritten as

$$
\max _{p} \pi\left(\left(q_{s}, p\right) \mid \theta_{j}\right)=\max _{p}\left[\alpha_{j} \cdot K(1-p)+2 \cdot K(1-p) \cdot(p-c)\right] .
$$

Therefore, formula (14) can be expressed as follows

$$
c=\left\{\begin{array}{ll}
p+\frac{\left(n \alpha_{j}+\alpha_{j} \cdot(m-1) / 2\right) \cdot(1-p)^{n}+(p-1)}{n+m-1}, & 0<p \leq 1 \\
p+\frac{p+\alpha_{j} \cdot(m-1) / 2-1}{m-1}, & \frac{-\alpha_{j}}{2}<p \leq 0
\end{array} .\right.
$$

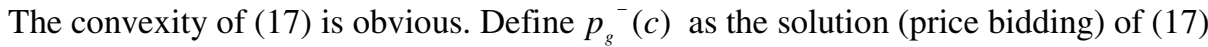
in the domain $p \in[-1,0]$.

Since the domain of expected revenue (16) is a closed set, in order to find the global maximizer, what we should do is to compare the boundary solutions with interior solution. Next, define $c^{*}$ as follows,

$$
c^{*}=\max \left\{c: \pi(0 \mid c)-\pi\left(p_{g}{ }^{*}(c) \mid c\right) \geq 0\right\} .
$$

Because

$$
\frac{d\left\{\pi(0 \mid c)-\pi\left(p_{g}{ }^{*}(c) \mid c\right)\right\}}{d c}=-2 K(1)+2 L\left(1-p_{g}{ }^{*}(c)\right) \cdot K\left(1-p_{g}{ }^{*}(c)\right)<0 .
$$

It follows that if there exits $c^{*}$, such that

$$
\pi\left(0 \mid c^{*}\right)-\pi\left(p_{g}^{*}\left(c^{*}\right) \mid c^{*}\right)=0 .
$$

then for all $c: 0<c<c^{*}$, we have

$$
\pi(0 \mid c)>\pi\left(p_{g}{ }^{*}(c) \mid c\right) .
$$

Define another special cost point $c^{-}$as follows

$$
c^{-}=\max \left\{c: \pi\left({p_{g}}^{-}(c) \mid c\right)-\pi(0 \mid c) \geq 0\right\} .
$$


Because

$$
\frac{d\left\{\pi\left(p_{g}{ }^{-}(c) \mid c\right)-\pi(0 \mid c)\right\}}{d c}=-2 K\left(1-p_{g}{ }^{-}(c)\right)+2 K(1)<0
$$

It follows that if there exits $c^{-}$, such that

$$
\pi\left(0 \mid c^{-}\right)-\pi\left(p_{g}^{-}\left(c^{-}\right) \mid c^{-}\right)=0 .
$$

Then for all $c: 0<c<c^{-}$, we have

$$
\pi(0 \mid c)<\pi\left(p_{g}{ }^{-}(c) \mid c\right) .
$$

Basically, in order to compare boundary solutions with interior solutions, we defined $c^{*}$ and $c^{-}$respectively. In other words, $c^{*}$ is the separating cost point below which zero price bidding is better than positive interior solution, while $c^{-}$is the separating cost point below which negative price bidding is better than zero price bidding. The following result is immediate.

Theorem 1. The following constitutes an equilibrium of MAPCA.

a) The simple bidder with type $\theta_{i}$ follows the strategy as (4);

b) The diverse bidder with type $\theta_{j}$ follows the strategies:

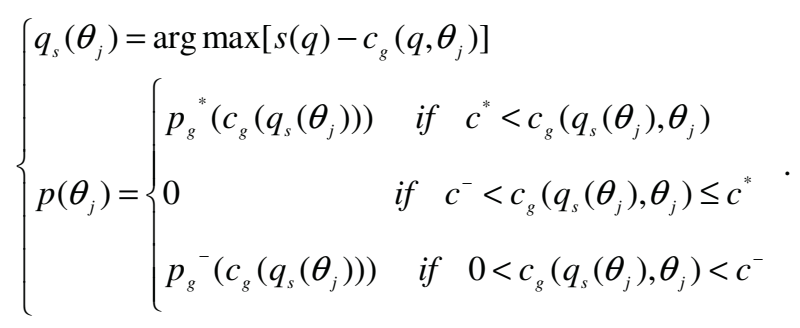

Intuitively, theorem 1 illustrates that one bidder either with synergy effect or not will submit same flavor quality-bid, i.e., optimal quality to maximize the difference of quality score minus the corresponding cost. As to price bidding, two kinds of bidders are apt to bid lower price when they have lower optimal cost. However, there exists two special cost "jump" points to make diverse bidders bid relative lower price: zero price or negative price. It is not so strange if we consider the situation in which synergy effect is very strong for any specific diverse bidder. The synergy effect can be regarded as business reputation, advertisement considerations and market share competition, which depends on different business surroundings.

\section{Conclusions}

In this paper, we construct a multi-attributes procurement combinatorial auction (MAPCA) for 2 identical objects, through a first-score, sealed-bid, simultaneous format. There are two kinds of bidders, simple bidder and diverse bidder, are considered in our model. With some assumptions, we obtain the equilibrium bidding strategies 
for the two kinds of bidders. Basically, the difference between our work and past correlated research (Krishna and Rosenthal (1996)) are: multi-attributes vs. single attribute, first-score vs. second-price, and procurement auction vs. selling auction. Also, this piece of research can not be realized without Che's work (Che 1993) about one-unit multi-attributes procurement auction. Compared with previous work about multi-attributes procurement combinatorial auctions' bidding analysis (Chen and Huang (2004)), this model extends to multiple diverse bidders who have synergy effect in the auction. Future work includes extensions to heterogeneous items assumption of our model.

\section{Acknowledgements}

This paper was partly supported by National Science Foundation of China under grant 70231010 and 70321001.

\section{References}

1. Che, Y. K.: Design competition through multidimensional auctions. The Rand Journal of Economics.Vol.24 (1993) 668-681

2. Chen J., H. Huang: Bidding Strategies Analysis for Procurement Combinatorial Auctions. Proceedings of The Fourth International Conference on Electronic Business. (2004) 41-45

3. Elmaghraby, W., P. Keskinocak: Technology for transportation bidding at the Home Depot. C. Billington, T. Harrison, H. Lee, J. Neale, eds. The Practice of Supply Chain Management: Where Theory and Applications Converge. Kluwer. (2003)

4. Hohner, G., J. Rich, E. Ng, G. Reid, A. J. Davenport, J. R. Kalagnanam, H. S. Lee, C.: An. Combinatorial and quality-discount procurement auctions with mutual benefits at Mars Incorporated. Interface. 33(1), (2003) 23-35

5. Krishna, V., Rosenthal, R.W.: Simultaneous Auctions with Synergies. Games and Economic Behavior, Vol. 17, (1996) 1-31

6. Ledyard, J. O., M. Olson, D. Porter, J. A. Swanson, D. P. Torma: The first use of a combined value auction for transportation services. Interfaces, 32(5), (2002) 4-12

7. Parkes, D.: Iterative Combinatorial Auctions: Achieving Economic and Computational Efficiency. Doctoral thesis, University of Pennsylvania (2001)

8. Rosenthal, R.W., Wang, R. Q.: Simultaneous Auctions with Synergies and Common Values. Games and Economic Behavior, Vol.17 (1996) 32-55 